\title{
ERJ
}

Engineering Research Journal

Faculty of Engineering

Menoufia University

\section{REVIEW OF SCADA SYSTEM FOR DISTRIBUTION POWER SYSTEM AUTOMATION}

\author{
A. A. Abou El-Ela \\ Electrical Engineering Department \\ Faculty of Engineering, Menoufiya \\ University \\ Egypt \\ draaa50@hotmail.com
}

\author{
Ragab A.El-Sehiemy \\ Electrical Engineering Department \\ Faculty of Engineering, Kafrelsheikh \\ University \\ Egypt \\ elsehiemy@eng.kfs.edu.eg
}

\author{
Ahmed M El-Shebiny \\ South Delta Electricity \\ Distribution Company (SDEDCo) \\ Ministry of Electricity, El \\ Gharbeya \\ Egypt \\ a_m_elshebiny@hotmail.com
}

\begin{abstract}
:
Power system should be economic, reliable, stable and with high quality operation of the system. The major challenge is to achieve its operation goals with overcome on its continuous problems, therefore power system automation comes into consideration to achieve the best quality operation level of electrical power system network. Automation is defined as the usage of machines thus eliminating human efforts to finish a task. Power system automation is the act of automatically monitoring and controlling the power system via instrumentation and control devices. Power system automation is to incessant monitoring and controlling of all elements and parts in the electrical network, this concept achieves by collecting all required and essential information from field to central monitor and control location. This paper provides an overview of the automation of the distribution power system architecture and its main components.
\end{abstract}

Index Terms - SCADA, IED, Automation, Distribution Automation, Communication.

\section{INTRODUCTION}

A distribution power system is to provide power to individual consumers and must be able to meet reasonable power demands by large and small customers. Electric utilities facing many problems in operating the distribution power network, most important is to reduce number of faults and minimize the outage period.

To overcome the network operation problems and increase its reliability, continuously network monitoring and controlling should be applied. Power system automation depends on collecting information and taking decisions by using devices which providing the essential information from the network and in the same time can control the network elements. Distribution automation gives the ability for network reconfiguration, fault identification, fault isolation, services restoration and avoid overloading.

Distribution power system definition is set of technologies that enable an electric utility to remotely monitor, coordinate and operate distribution components in a real-time mode from remote locations.

Distribution power system automation uses Supervisory Control and Data Acquisition system (SCADA) to achieve the automation concepts and goals. SCADA is a control system used on geographically wide area network which is appropriate for the distribution power system. SCADA system is a complete control system applied on the substation level plus the network level by using communications system.

A. Distribution Automation principles

Distribution automation is to gathering all necessary data from the network to control center using SCADA.
The SCADA system is stands for Supervisory Control and Data Acquisition system, which is depend on:

- Collecting data and information

- Data acquisition and analysis

- Network operation and supervision

- Controlling

Data is the basis of the control center which is giving the required information to operator to take the right decision in network operation. Many types of data are transferred to control center, like elements status and conditions, sequence of events and protection functions alarms. Another type of data is the measurements which is monitored instantaneous like voltage, current, active power, reactive power and power factor. Transferring all measurements to the control center prevent engineer from site attendance and provide all required data for network study.

After collecting all types of data and stored in the control center, many applications available in the SCADA system which are performing analysis and studies which help the operator and planning engineer to take the right decisions for operating the network.

One of the important functions of SCADA is that gives the ability to control the different elements of the power network system which help in quickly fault isolation and service restoration.

\section{B. Distribution Automation Features}

Distribution companies implementing distribution automation (DA) are receiving benefits from many areas such as providing a fast method of improving reliability, making 
the whole operating function more efficient, or simply extending asset life.

Distribution automation provides many features, which include, improved public image, full information, increase system reliability, increase system efficiency, customer satisfaction, reduce expenses, modern technology and smart operation. [1].

Several supporting functions assist in achieving the main distribution automation system features and objectives, including the following:

- Metering and control

- Fault locating

- Fault isolation

- Safety checks

- Operating constraint checks

- Topology analysis

- Reliability calculations and logging

- Event recording

- Visualization

- Communications monitoring. [2].

\section{SCADA SYSTEM ARCHITECTURE}

SCADA is an integrated control system consisting of a number of homogeneous stations connected by a communications network. [3].

Three main categorizes forms the architecture of the SCADA system:

- Substation Automation

- Communication Network System

- Master Station

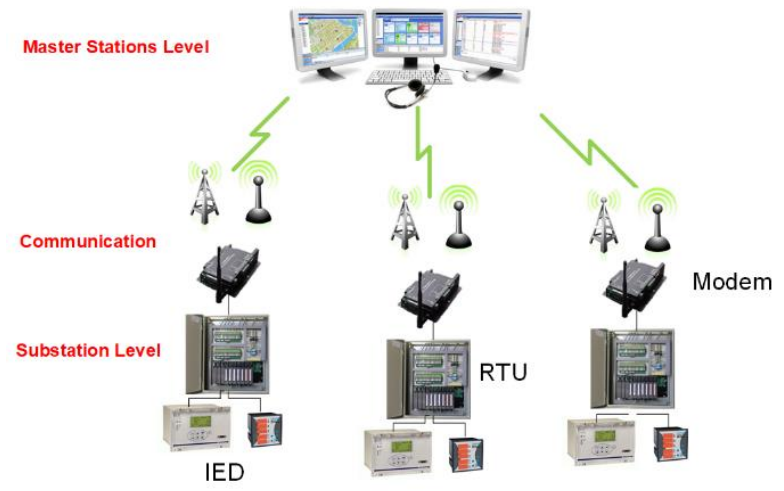

Figure 1 SCADA System Architecture

A. Substation Automation

Basically the substation automation level consists of sublevels,

- $\quad$ Level 1 (Field / Process):

This level contains the field equipment and Switch gear, CTs, PTS, etc. Monitoring and measurement of system parameters are carried out at this layer.

- Level 2 (Bay):

This level contains the protection and control equipment. Protective relays, RTUs and IEDs constitute this level. The collected information for level 1 is processed to this level.
- Level 3 (Station):

This level contains the operator display and engineering workstation for executing the programs and monitoring \& controlling the substation from central location.

There are two main types of automation for the substation. The first type is the RTU (Remote Terminal Unit) based solution. RTU is a device installed at a remote location that collects data, codes the data into a format that is transmittable and transmits the data back to the control center. RTUs are equipped with input channels for sensing, metering, indication or alarms and with output channels for control and communications ports.

This type is based on connecting the field signals through hardwire connection to the interface cards in the RTU plus connection through protocol to the protection relays for the protection signals and another connection to the MFM (MultiFunction Meter) for transferring the measurements.

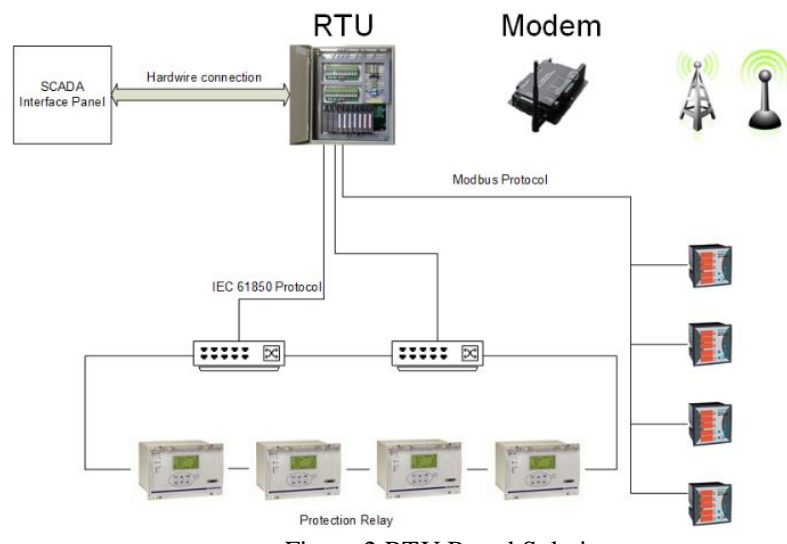

Figure 2 RTU Based Solution

The second type is the SCS (Substation Control System) which based on installing BCU (Bay Control Unit) gathering all signals types from a feeder and then all BCUs are connected through communication LAN to the central servers $\&$ gateways in the station.

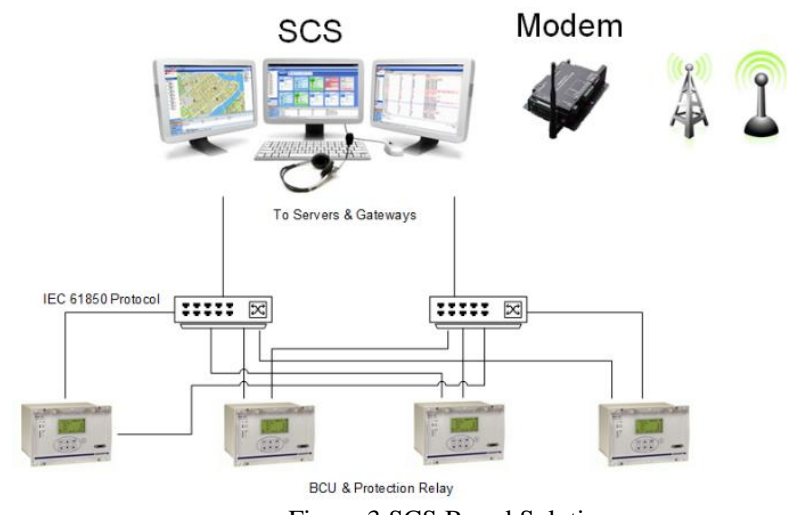

Figure 3 SCS Based Solution 


\section{B. Communication Network System}

The nerve connecting all parts of the system is the communication network. Without a properly designed and reliable communication network system, a SCADA system cannot exist. All supervisory control and data acquisition aspects of the SCADA system rely entirely on the communication system to provide a conduit for flow of data between the supervisory controls, the data acquisition units, and any controllers that may be linked to the system. The purpose of a communications network within a SCADA system is to connect the remote terminal units (RTUs) / SCS Gateways with the SCADA Master Station.

Two main types for the communication system:

1- Wired: Many options for the wired communication system like, Telephone Line, Ethernet, Power Line Carrier, Fiber Optic and Coaxial Cable.

2- Wireless: Different types for the wireless communication system like, UHF, VHF, Wi-Fi, Microwave, Cellular and Satellite.

\section{Master Station}

Master station forms the virtual brain of the power system automation system. The SCADA master station receives data and information from the field, decides what to do with it. Master station consists of multiple servers and software applications.

In modern systems, the Master Station is typically a collection of computers, peripherals, and appropriate input I output (I/0) subsystems that enable the dispatchers to monitor the state of the plant, say, Power system and to control it. Master station equipment enables the dispatcher to monitor the system: visually, by audible alarm, and by hard copy. [4].

The SCADA master station consists of two parts:

\section{1- Servers Room:}

Location which housing all types of servers and devices like, Front-End servers, data engineering servers, SCADA servers, communication LAN, external disk storage and GPS. All these devices responsible for interfacing to the outstation through the communication cloud.

2- Dispatcher Room:

It is contains workstations with HMI (Human Machine Interface) which enable the control engineer to monitor and control the network. Operator workstation, system administration \& training workstation, engineering workstation and printers are all devices available in the dispatcher room.

An important aspect of every SCADA system is the computer software used within the system. The most obvious software component is the operator interface or Human Machine Interface (HMI) package. The software computers process the information received from and sent to the RTU sites and present it to human operators in a form that the operators can work with. The software is programmed to tell the system what and when to monitor, what parameter ranges are acceptable, and what response to initiate when parameters change outside acceptable values.
SCADA System Master Stations are vital to safe, reliable, and economic operation of utility systems. Interruptions to the operation of the critical functions that they perform must therefore be as infrequent as possible. Moreover, whenever a failure occurs, the system must be repaired in minimum time.

\section{SCADA COMMUNICATION PROTOCOLS}

In SCADA system, data flow between RTUs in the remote station and the master station (Control Center). The data representations sent are not identified in any fashion other than by unique addressing. The addressing is designed to correlate with the SCADA master station database. The RTU/SCS has no knowledge of which unique parameters it is monitoring in the real world. It simply monitors certain points and stores the information in a local addressing scheme. [5].

SCADA system used multiple communication protocols to enable data transferring between remote station and master station. The most common protocols used in SCADA system are:

- IEC 60870-5-101

- Distributed Network Protocol version 3 (DNP3)

- IEC 60870-5-104

- Modbus

Each protocol has its own features and advantages and it is important when applying SCADA system to choose the suitable communication protocol.

On the other hand, there are many communication protocols used for data communication between the substation control level like protection relay, MFM and BCU and the RTU/SCS. Most advanced and modern protocol used for IED is IEC61850 which is an adequate solution to provide real-time information to the distribution automation control center, allowing it to perform a fast supply and demand management. [6].

\section{SCADA APPLICATIONS PROTOCOLS}

Based on data received from SCADA and to achieve the data acquisition goal, many SCADA applications could be applied to provide the required and essential data and information to the operation and planning.

Distribution management systems include the real-time functionalities of distribution SCADA coupled with the relevant application functions with support from the corporate process systems such as customer information systems (CISs) and geographical information systems (GISs). DMSs are also integrated with outage management systems (OMSs) and asset management systems (AMSs).

There are many considerations on how to control so much data. There is the centralized solution of DMS. A DMS is a real-time information system for all operational activities in a modern distribution control room. [7].

Distribution Management System is including multiple applications installed on the SCADA platform [8], like: 
- Advanced alarms management

- Network management

- Fault management

- Outage report

- Switching orders

- Network simulation

- Distribution feeder optimization

- Load flow analysis

- Short-circuit calculator

- Protection coordination

- Automatic voltage regulator (AVR) application

- Load forecast

- Fault detection, isolation, and service restoration (FDIR)

With all the aforementioned elements in place plus that crucial two-way communication with the customer, utilities can improve their reliability indices and begin to engage customers in a virtual cycle that further contributes to speedier outage detection, power restoration, and customer satisfaction.

\section{DISTRIBUTION AUTOMATION KEY DEVICES}

Distribution automation and SCADA system hinge on the information and data received from remote stations, based on that, the need to have devices in remote stations to send and transfer its data from field to control center is become essential for automation.

The foundation of the monitoring system infrastructure is based on sensors, transducers and intelligent electronic devices (IED) collecting information throughout the distribution system. [9]

The most important element which responsible to most of data received by control center is the intelligent Electronic Device (IED). There are many types of smart grid devices which install in the station based on the functions required, such as:

\section{A. Smart Meters}

Smart meters are capable of measuring instantaneous voltage and instantaneous current of the electrical circuit it connected to. Digital circuit in the meter, having a processor inside can calculate any other parameter like instantaneous power, total energy usage, power factor and many other parameters. [10]

Smart meters can enhance the operation of SCADA system. As smart meter system provides several benefits such as efficient power system control and monitoring, operational decisions those are taken timely to minimize outages and losses. In smart grid, smart meters can perform energy cost allocation, fault analysis, demand control and power quality analysis. Smart meters can schedule preventive maintenance, and support the operation of check meters for accurate billing. In addition, smart meters can detect the presence of unwanted harmonic component in current supplied from the de-centrally generated sources, which helps in identification and rectification of the source of the problem. [11]

\section{B. Bay Control Unit (BCU)}

Bay Control Unit (BCU), is a highly versatile panelmounted unit providing a wider range of control and automation capabilities at the individual bay. The BCU has a powerful user interface that can present single line diagrams, status, alarms and measurements. It is a key element within the flexible Substation Automation System.

In addition to the monitoring functions, $\mathrm{BCU}$ also support all control functions that are required for operating the station. With integrated logic, the user can set, via a graphic interface (CFC), specific functions for the automation of switchgear or substation. Functions are activated via function keys, binary input or via communication interface. [12]

The BCUs and other smart devices using many communication protocols, IEC 61850 is widely used. IEC 61850 has a significant impact on all aspects of the Smart Grid and is defined as one of the key technologies for its development and implementation. [13]

\section{RTU \& MEASURMENTS ALLOCATION APPROACHES}

The allocation design of RTUs and measurements devices like PMU in the distribution network is essential to reduce the numbers of RTUs \& PMUs which reflect to reduce the cost.

There are two main constrains forming the allocations design which are, maintaining observability and minimizing number of equipments. [14]

Since the RTU is one of the main key devices in the automation of the distribution network and the ways for allocation of it in the network is differs based on many aspects, different approaches for allocation of RTU is presented such as,

- RTU Placement Procedure Using Fuzzy Sets Theory, which is a qualitative-quantitative approach by taking Analytical Hierarchical Process (AHP) as the basis. [15]

- Remote terminal unit (RTU) placement algorithm which is to place a minimum set of RTUs which satisfies three important constraints of the problem- observability, absence of critical measurements, and reliability requirements. The results of this algorithm are interfaced with a linear programming (LP) state estimator. The RTU placement algorithm and its link with the LP estimator show that the LP estimator has very good filtering capabilities when operated with or without bad data, and that the loss of any RTU does not have any significant impact on the solution. [16] 


\section{SCADA CYBER SECURITY}

The increasing interconnectivity of SCADA (Supervisory Control and Data Acquisition) networks has exposed them to a wide range of network security problems. There is now an increased interest in strengthening industrial cyber security.

Three challenges must be addressed to strengthen SCADA networks. The first challenge is to improve the access controls to the SCADA networks. A solution will make it harder for an attacker to enter into the SCADA network. The second challenge is to improve security inside the SCADA network and to develop efficient security-monitoring tools. The security mechanisms developed to address this challenge will ensure that even if an attacker manages to enter the SCADA network, it will be difficult to carry out any sort of attack. The monitoring tools will help to detect intrusions and other suspicious activities on the network. The third challenge is to improve the security management of the SCADA network. [17].

There are three security goals to be achieved in SCADA system, first is Service Integrity which defined as the trustworthiness of the information provided by the sensor network. The quality of the received information allows the sensor network to perform its intended function, which is the collection of accurate data from the sensors. Second goal is Network Availability which is the information collected by the sensors is accessible and useable upon demand by a legitimate user. A violation of network availability results in denial of service: the prevention of authorized access to the sensor measurements. Third goal is Confidentiality and Privacy, Confidentiality: the information collected by sensor networks is only accessible to legitimate users. It is intended to prevent unauthorized users from learning the information collected by the sensors. A violation of confidentiality results in disclosure. Privacy is the prevention of unauthorized users from learning sensitive personal information by using the sensor network. [18].

There are different types of threats such as Terrorist, Insider, Hacker and Malware in SCADA systems. [19].

In general, almost all SCADA systems besides IT systems' vulnerabilities, threats and risks, they have their own software and hardware vulnerabilities and weaknesses. Therefore SCADA systems owners should replace their SCADA devices and networks with secure systems. These transformations need to be concerned in different area such as owners' awareness about the SCADA systems security and implementation of the SCADA systems' standards and keep the systems maintained base on the standards. Based on the vulnerabilities and the SCADA attacks, some prevention methods such as penetration testing, Attack Mitigation, SCADA Honeynet Systems and Preventative Systems are proposed as solutions.

The benefits from applying distribution automation could be categorized to three main areas as follows: [20].

- Financial benefits:

- Deferral of capital investment (new TS, additional transformer capacity, additional feeders,
- Increased revenue due to quick restoration

- Reduced operation and maintenance cost

- Improved utilization of system capacity

- Reduced system loss

- Customer retention for improved quality of supply

- Operational \& Maintenance:

- Improved reliability by reducing outage duration

- Improved voltage control

- Reduced man hour and man power

- Accurate and useful planning and operational data / information

- Better fault detection and diagnostic analysis

- Better management of system and component loading

- Customer related benefits:

- Better service reliability

- Reduce interruption cost for customers

- Better quality of supply

In addition, distribution automation improves reliability and its indices, SAIDI, SAIFI \& CAIDI. [21].

\section{CONCLUSION}

In this paper, review on distribution power system automation techniques. Supervisory Control and Data Acquisition System (SCADA) is the control system which is implemented in distribution power system with many different techniques and designs.

SCADA system has its own features and advantages which can effectively decrease the number of customer minutes lost and provide other network management benefits, such as reducing the cost of ownership and improving the customer interface, but the benefits must be balanced against the cost and this is the most challenge point in the distribution automation, the cost benefits against implement the distribution automation.

Distribution automation and system monitoring are the logical choice for the utilities to improve system performance, and to achieve customers and shareholders satisfactions. 


\section{REFERENCES}

[1] Ankita Pai and, Tejas Kopte, "A Study on power system automation", IJAREEIE, Vol. 4, Issue 9, September 2015.

[2] Richard Greer, Will Allen, Jim Schnegg, and Andrew Dulmage, "Distribution automation systems with advanced features", 2010 by American Electric Power and Schweitzer Engineering Laboratories, Inc.

[3] Branislav Atlagiü, Mihalj Šagi, "Proposal of a modern SCADA system architecture", 19th Telecommunications forum TELFOR 2011, Serbia, Belgrade, November 22-24, 2011.

[4] Tina Agarwal and Zeida Fatima, "Master station architecture of a scada system," IETE Journal of Education Vol43, No 3, July-September 2002, pp. 121-126.

[5] National Communication System," Supervisory control and data acquisition (scada) systems", NCS TIB 04-1, October 2004.

[6] Quentin Morel and Thierry Coste, 'IEC 61850 to the service of power system flexibility", 24th International Conference \& Exhibition on Electricity Distribution (CIRED), 2017, Vol. 2017, Iss. 1, pp. 1792-1794.

[7] Roop, David W, "Power system scada and smart grids book"

[8] Nikos Hatziargyriou1 $\square$, Vasilis Kleftakis2, Foivos Palaiogiannis2, Aris Dimeas2, Ioannis Vlachos2, Christos Katsanos1, Asterios Zafeirakis1, Andreas Tzevelekos1, "Towards the modernisation of the supervisory control and data acquisition systems of the hellenic electricity distribution network operator: considerations and steps forward", CIRED, Open Access Proc. J., 2017, Vol. 2017, Iss. 1, pp. 1516-1519.

[9] Francisc zavoda, "The key role of intelligent electronic devices (led) in advanced distribution automation (ada)", CICED2008 Technical Session 3, 83-26 FP1366.

[10] N. Athula. Kulatunga, IEEE Member, Sudheera Navaratne, Jeremiah Dole, Chamika Liyanagedera, and Tom Martin, "Hardware development for smart meter based innovations", IEEE PES ISGT ASIA 2012 1569527895 .
[11] Soma Shekara Sreenadh Reddy Depuru, Lingfeng Wang*, Vijay Devabhaktuni, "Smart meters for power grid: challenges, issues, advantages and status", Renewable and Sustainable Energy Reviews 15 (2011) 2736-2742

[12] Azza Siraj El-Dien M. El-Amien, "Design of a bay control unit in a substation automation system", Department of Electrical And Electronic Engineering Faculty of Engineering University of Khartoum, July 2009

[13] A. P. Apostolov, Fellow, IEEE, "IEC 61850 based components, interfaces and services for a smart grid".

[14] Garng M. Huang, Jiansheng Lei, "A Topological measurements and rtus design against a contingency", V-920, IEEE 2004.

[15] Payman Dehghanian, Ali Asghar Razi Kazer, and Ghasem Karar, "Incorporating experts knowledge in rtu placement procedure using fuzzy sets theorya practical approach", 978-1-4577-1250-0/11 @2011 IEEE

[16] Mounir Yehia, Member, IEEE, Rabih Jabr, Ibrahim El-Bitar, and Richard Waked, "A PC based state estimator interfaced with a remote terminal unit placement algorithm", IEEE TRANSACTIONS ON POWER SYSTEMS, VOL. 16, NO. 2, MAY 2001

[17] Vinay M. Igure, Sean A. Laughter, Ronald D. Williams, "Security issues in SCADA networks, computers \& security", 25 (2006) 498-506

[18] Alvaro A. Cardenas , Tanya Roosta, Shankar Sastry, "Rethinking security properties, threat models, and the design space in sensor networks: A case study in SCADA systems", Ad Hoc Networks 7 (2009) 1434-1447

[19] Farid Daryabar, Ali Dehghantanha , Nur Izura Udzir , Nor Fazlida binti Mohd Sani and Solahuddin bin Shamsuddin, "Towards secure model for scada systems"

[20] Rong-Liang Chen, Shafi Sabir, "The benefits of implementing distribution automation and system monitoring in the open electricity market"

[21] Yeliz Yoldas, Ahmet Onen, Irfan Alan, Robert P. Broadwater, "Distribution automation effects on reliability during major contingencies" 